\title{
Cingulum stimulation enhances positive affect and anxiolysis to facilitate awake craniotomy
}

\author{
Kelly R. Bijanki, ${ }^{1,2}$ Joseph R. Manns, ${ }^{3}$ Cory S. Inman, ${ }^{1}$ Ki Sueng Choi, ${ }^{2}$ Sahar Harati, ${ }^{4}$ Nigel P. Pedersen, ${ }^{5}$ Daniel L. Drane, ${ }^{5}$ \\ Allison C. Waters, ${ }^{2}$ Rebecca E. Fasano, ${ }^{5}$ Helen S. Mayberg, ${ }^{2,5}$ and Jon T. Willie ${ }^{1,5}$ \\ 'Department of Neurosurgery, and 'Department of Psychiatry, Emory University School of Medicine, Atlanta, Georgia, USA. ${ }^{3}$ Department of Psychology, Emory University College of Arts and Sciences, \\ Atlanta, Georgia, USA. ${ }^{4}$ Department of Biomedical Informatics, and ${ }^{5}$ Department of Neurology, Emory University School of Medicine, Atlanta, Georgia, USA.
}

\begin{abstract}
BACKGROUND. Awake neurosurgery requires patients to converse and respond to visual or verbal prompts to identify and protect brain tissue supporting essential functions such as language, primary sensory modalities, and motor function. These procedures can be poorly tolerated because of patient anxiety, yet acute anxiolytic medications typically cause sedation and impair cortical function.
\end{abstract}

\begin{abstract}
METHODS. In this study, direct electrical stimulation of the left dorsal anterior cingulum bundle was discovered to reliably evoke positive affect and anxiolysis without sedation in a patient with epilepsy undergoing research testing during standard inpatient intracranial electrode monitoring. These effects were quantified using subjective and objective behavioral measures, and stimulation was found to evoke robust changes in local and distant neural activity.

RESULTS. The index patient ultimately required an awake craniotomy procedure to confirm safe resection margins in the treatment of her epilepsy. During the procedure, cingulum bundle stimulation enhanced positive affect and reduced the patient's anxiety to the point that intravenous anesthetic/anxiolytic medications were discontinued and cognitive testing was completed. Behavioral responses were subsequently replicated in 2 patients with anatomically similar electrode placements localized to an approximately $1-\mathrm{cm}$ span along the anterior dorsal cingulum bundle above genu of the corpus callosum.
\end{abstract}

CONCLUSIONS. The current study demonstrates a robust anxiolytic response to cingulum bundle stimulation in 3 patients with epilepsy.

TRIAL REGISTRATION. The current study was not affiliated with any formal clinical trial.

FUNDING. This project was supported by the American Foundation for Suicide Prevention and the NIH.

\section{Introduction}

Neurosurgical procedures are performed in awake patients when evaluation of neurological function is necessary. Typical examples include deep brain stimulation (DBS) electrode placement for movement disorders and awake craniotomy to resect mass lesions or seizure foci near "eloquent" cortices, which, if removed, can cause obvious motor, sensory, or linguistic disability. When patients are too anxious to tolerate awake procedures, surgery under general anesthesia risks failure to achieve maximal safe

\section{Related Commentary: p. 952}

Conflict of interest: KRB, JTW, NPP, and CSI are named inventors on a patent filing based on the work described herein (US Patent Application no. 16121599 [pending]: A method of electrically stimulating the dorsal anterior cingulum bundle to reduce anxiety, reduce pain, facilitate cognitive performance, and elicit spontaneous laughter, smiling, and euphoria). HSM receives fees from the licensing of intellectual property to St. Jude Medical Inc.; DLD receives grant funding from Medtronic Inc. and honoraria from NeuroPace Inc.; and JTW serves as a consultant for Medtronic Inc., MRI Interventions Inc., and NeuroPace Inc. License: Copyright 2019, American Society for Clinical Investigation.

Submitted: January 22, 2018; Accepted: December 18, 2018.

Reference information: / Clin Invest. 2019;129(3):1152-1166. https://doi.org/10.1172/JCI120110. resection with minimal collateral disability. A nonsedating method that facilitates patient tolerance of awake functional-anatomical correlation would be potentially transformative for neurosurgery.

The cingulum bundle is a white matter tract coursing longitudinally from anterior to posterior, wrapping around the corpus callosum from genu to splenium. The cingulum bundle serves to connect the cingulate cortices, dorsomedial prefrontal cortices, basal forebrain, parietal precunei, and temporal hippocampal/ parahippocampal gyri (1-3). The anterior cingulate cortex (ACC) has been ascribed putative roles in a wide variety of emotional and cognitive functions, including modulation of affective responses, error detection and conflict monitoring, regulation of anxiety and depression, attention to physical pain and social distress, and the motivation to persevere (4-7). Electrical stimulation of dorsal ACC gray matter can elicit transient euphoria and/ or analgesia (8-12), and the cingulum bundle has recently been suggested to mediate part of the therapeutic effects of DBS for depression $(13,14)$. Indeed, white matter stimulation is increasingly recognized as a preferred approach to therapy, especially for psychiatric disorders (15-17).

Patients with focal medically refractory epilepsy often undergo diagnostic intracranial electroencephalography (iEEG) 
with temporarily implanted electrode arrays to localize seizure onsets in advance of definitive surgical therapies (18). We report the case of a patient with epilepsy undergoing iEEG that included depth electrodes within the anterior cingulum bundle. Low-amplitude stimulation induced acute outward signs of mirth, subjective reports of happiness and relaxation, and persistent objective behavioral features of positive affect. After characterization of acute behavioral and electrophysiological responses, prolonged cingulum stimulation provided robust and effective anxiolysis without sedation during awake resection for epilepsy in the language-dominant posterior temporal lobe. Similarly low-threshold behavioral responses to stimulation were replicated in 2 additional epilepsy iEEG patients with very similar electrode placement in the cingulum bundle. Cingulum bundle stimulation may have implications for the treatment of anxiety and mood disorders.

\section{Results}

\section{Part I: Research study of affective modulation via limbic stimulation} Case history and clinical characteristics of the index patient. A 23-year-old left-handed, English-speaking woman with an 8-year history of chronic medically refractory focal dyscognitive seizures underwent comprehensive epilepsy evaluation that included scalp EEG with poorly localized left hemispheric seizure onsets, normal MRI with left hemispheric language dominance, left temporal interictal hypometabolism on 5-deoxyglucose PET, magnetoencephalogram with few poorly developed epileptiform discharges in the posterior temporal region, and nonlateralizing neuropsychological findings with normal intelligence (for additional clinical characteristics, see the Supplemental Materials, which are available online with this article; https://doi.org/10.1172/ JCI120110DS1). Prior intracranial electrode monitoring at another institution localized seizures in part to the left posterior temporal-parietal junction near sensory cortices and Wernicke's area, but resection had not been performed.

In addition to epilepsy, the patient had a history of untreated mild depression that was attributed to the side effects of antiepileptic medications. Standard neuropsychological measures revealed only minimal depression (Beck Depression Inventory $[\mathrm{BDI}]=4$ ) but moderate anxiety (Beck Anxiety Inventory [BAI] = 22) at baseline, indicating a potentially clinically significant symptom burden (19).

Behavioral replication. Initial findings were replicated in 2 additional iEEG patients. Patient 2 was a 40-year-old left-handed, English-speaking man with a history of treatment-refractory epilepsy since 8 years of age but no evidence of clinically significant depression or anxiety at baseline $(\mathrm{BDI}-\mathrm{II}=5, \mathrm{BAI}=3)$. Patient 3 was a 28 -year-old right-handed, English-speaking woman with a 20-year history of treatment-refractory epilepsy and a history of depression dating back to age 8 and for which she was treated during her teenage years with antidepressant medications and psychotherapy. Her self-reported depression was less severe at the time of evaluation, despite a slightly elevated measure (BDI-II = $11, \mathrm{BAI}=2$ ). Additional clinical details are presented in the Supplemental Materials. Neither patient 2 nor patient 3 underwent awake neurosurgery to treat their epilepsy.

\section{Localization of electrodes and volume of activation}

\section{in the cingulum bundle}

The index patient underwent implantation of subdural grid electrodes over the perisylvian regions of the left frontal, temporal, and parietal lobes, strip electrodes over the left lateral temporal and parieto-occipital cortices, and stereotactic depth electrode arrays targeting the bilateral temporal lobes (including lateral cortices, amygdalae, and hippocampi) and medial left frontal and parietal lobes (including left anterior, dorsal, and posterior cingulate cortices). This implantation schema was developed to further investigate the possible parietal onset, while also covering regions that were potentially either part of the ictal-onset zone or seizure network, based on semiology and other available clinical data. One depth electrode array passed from the left parietal lobe anteriorly and obliquely to run through the left dorsal anterior cingulate cortex and cingulum bundle, with contacts at 8-mm intervals. The patient was monitored in the inpatient epilepsy monitoring unit for 8 weeks prior to resection, owing to a low frequency of seizures after implantation.

Figure 1 shows the location of electrode contacts within the index patient's cingulum bundle. We coregistered the patient's volumetric imaging of electrodes relative to brain (MRI T1 sequence) to her diffusion-weighted imaging for tractography (combination of linear and nonlinear registration: FMRIB's Linear Image Registration Tool [FLIRT] and FMRIB's Nonlinear Image Registration Tool [FNIRT], FMRIB Software Library). Using standard methods for modeling the effects of DBS (20), we estimated the volume of tissue activated (VTA) by 1.5-mA stimulation at the applied parameters as spherical seeds for the stimulated bipolar contact pairs. We generated maps of white matter tracts intersected by the VTAs, revealing the most likely pathways of activation corresponding with each stimulated contact pair (contacts 1-2, 3-4, and 5-6). For each contact pair, anodal stimulation was modeled as $1.0-\mathrm{mm}$ radius spherical regions of interest, and cathodal stimulation was modeled as $1.5-\mathrm{mm}$ radius spherical regions. The contact pair that evoked the strongest positive behavioral response (contacts $3-4$ ) was found to engage exclusively and robustly the cingulum bundle (Figure 1E) and corresponded most closely with the second and third segments (CB-II and CB-III) of the cingulum bundle, which are described from diffusion-weighted imaging and postmortem anatomical studies (1). The CB-II segment courses from the medial aspect of the superior frontal gyrus, along the primary cingulum bundle, and curves around the splenium before branching at the back, with a major output to the parahippocampal gyrus (this termination is unable to be modeled in a single case, because the angle of bending of the fiber track precludes reliable parcellation). The CB-III segment is the largest of the fiber bundles in the cingulum system, encompassing a different set of fibers originating from the medial aspect of the superior frontal gyrus, joining the main cingulum bundle over the corpus callosum, and innervating the precuneus.

By comparison, VTA models for stimulating the anterior pair of contacts 1-2 (primarily cingulate gray matter) suggested only limited engagement of the cingulum bundle (Figure 1E) and were not associated with any patient-reported subjective effects. VTA models of the posterior cingulum bundle pair (contacts 5-6) predicted robust engagement not only of the cingulum bundle but 

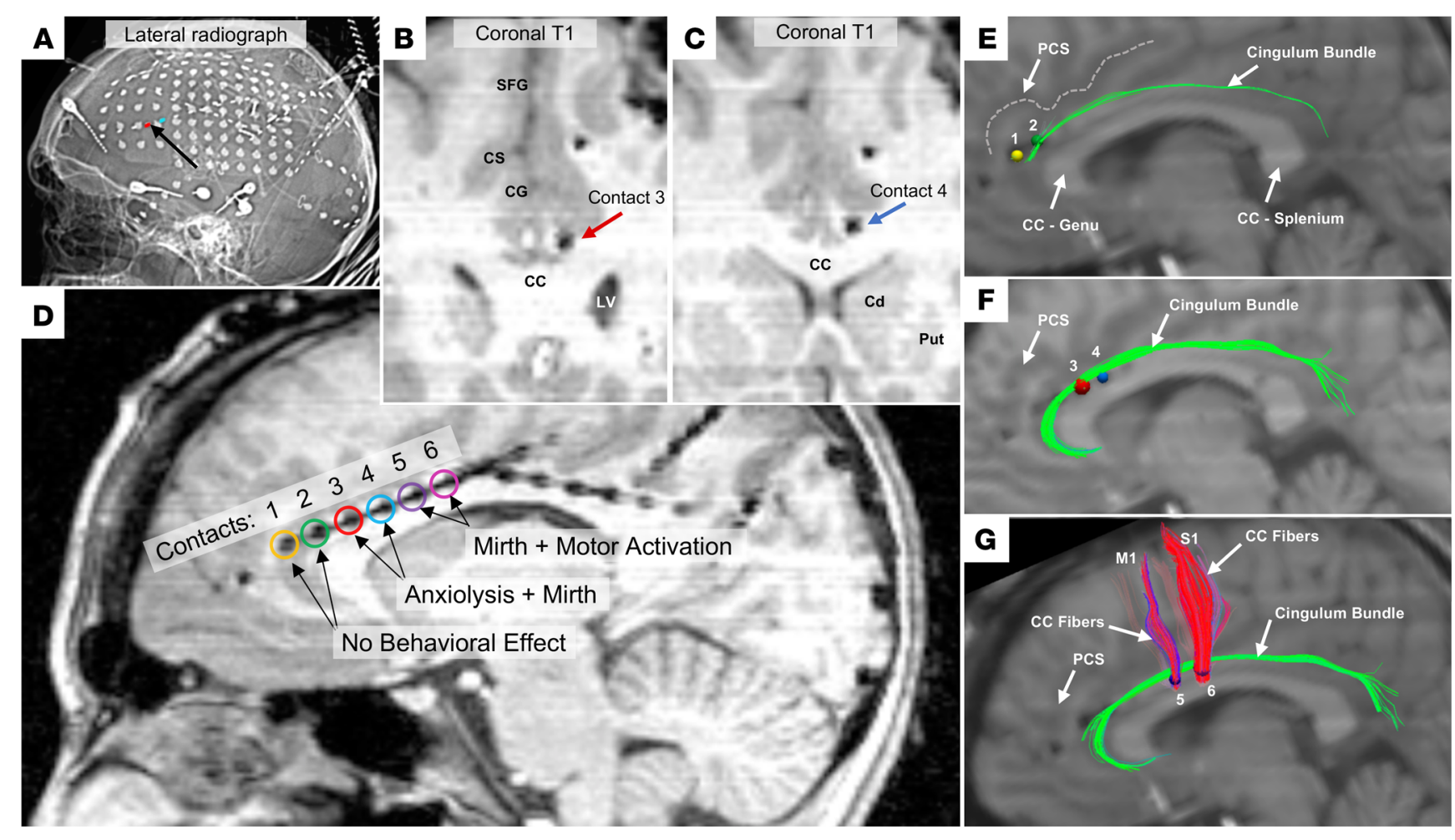

Figure 1. Location of stimulated electrodes in the index patient. (A) Postimplantation lateral skull radiograph showing relative positions of the intracranial electrodes; red and blue volumes indicated by a black arrow highlight the contact pair yielding anxiolytic benefit. (B-D) Postimplantation MRI demonstrating positions of stimulated cingulate contacts; coronal slices in B and C correspond to the anode (red) and cathode (blue) of stimulation-eliciting anxiolysis and mirth. Cingulate gray matter stimulation utilized contacts 1-2 (yellow, green), cingulum bundle stimulation eliciting anxiolysis and mirth utilized contacts 3-4 (red, blue), cingulum bundle stimulation eliciting mirth and motor activation utilized contacts 5-6 (purple, magenta). The recording site for single-channel and coherence electrophysiological analyses was contact 5 (purple). (E-G) Sagittal views of deterministic tractography seeded at all tested contact pairs using modeled bipolar volumes of tissue activated via artificial neural nets based on $1.5 \mathrm{~mA}, 130 \mathrm{~Hz}, 300-\mu \mathrm{s}$ pulse width stimulation. (E) Contacts 1 (anode; yellow) and 2 (cathode; green). (F) Contacts 3 (anode; red) and 4 (cathode; blue). (G) Contacts 5 (anode; purple) and 6 (cathode; pink). SFG, superior frontal gyrus; PCS, paracingulate sulcus, CG, cingulate gyrus; CC, corpus callosum; LV, lateral ventricle; Cd, caudate; Put, putamen; M1, primary motor cortex; S1, primary sensory cortex.

also of the superior aspect of the corpus callosum innervating primary motor and sensory cortices (Figure 1G). Indeed, stimulation of the posterior contact pair evoked not only mirth but also subjective motor activation (unpleasant neck pulling).

Two subsequent patients had extremely similar engagement of the cingulum bundle per VTA modeling conducted under the same assumptions as for the index patient (Figure 2). The anatomical localizations of all stimulated electrode contacts across the entire patient group $(n=3)$ are presented in Figure 2, which demonstrates tight clustering of behaviorally active contacts across the group. Individual-level details of cingulum bundle contact positions are presented in Supplemental Figures 1 and 2.

\section{Acute dorsal anterior cingulum stimulation elicited markers of positive affect}

We investigated whether electrical stimulation of so-called limbic system structures in the index patient elicited emotional responses for research purposes after clinical documentation of spontaneous seizures. First, we screened for any undesirable stimulation-induced after-discharges and established amplitude dose-response relationships for any subjective emotional or somatic responses (Figure 3). Stimulation was delivered in cur- rent-regulated, charge-balanced, biphasic symmetrical rectangular pulses using a neurostimulator for humans (CereStim R96, Blackrock Microsystems). Specifically, stimulation was performed in series of 5 -second-duration trials at $130-\mathrm{Hz}$ frequency $(300-\mu \mathrm{s}$ pulse width) and $50-\mathrm{Hz}$ frequency (200- $\mu$ s pulse width). Stimulation currents were increased in 0.5-mA steps from 0.5 to $3.5 \mathrm{~mA}$ (thus up to $17 \mu \mathrm{C} / \mathrm{cm}^{2} /$ phase, see Methods), with 3 trials at each current level (Table 1 and Supplemental Table 1). The following left hemispheric brain regions were stimulated: basolateral amygdala, dorsal anterior cingulate allocortex (gray matter), dorsal anterior cingulum bundle (white matter), dorsal mid-cingulum bundle, and the middle temporal gyrus (neocortical control). Only stimulation to the amygdala elicited asymptomatic epileptiform after-discharges and was therefore not continued. Stimulation to the amygdala, middle temporal gyrus, and anterior cingulate gray matter produced no subjective changes in mood, sensation, or motor function, as observed by the patient and the experimenters.

Stimulation to the left anterior cingulum bundle white matter (130 Hz, $300 \mu \mathrm{s}, 1.0 \mathrm{~mA}$ ) immediately elicited mirthful behavior, including smiling and laughing, and reports of positive emotional experience (Table 1, Table 2, and Figure 3). The patient described the experience as pleasant and relaxing and completely unlike any 
A

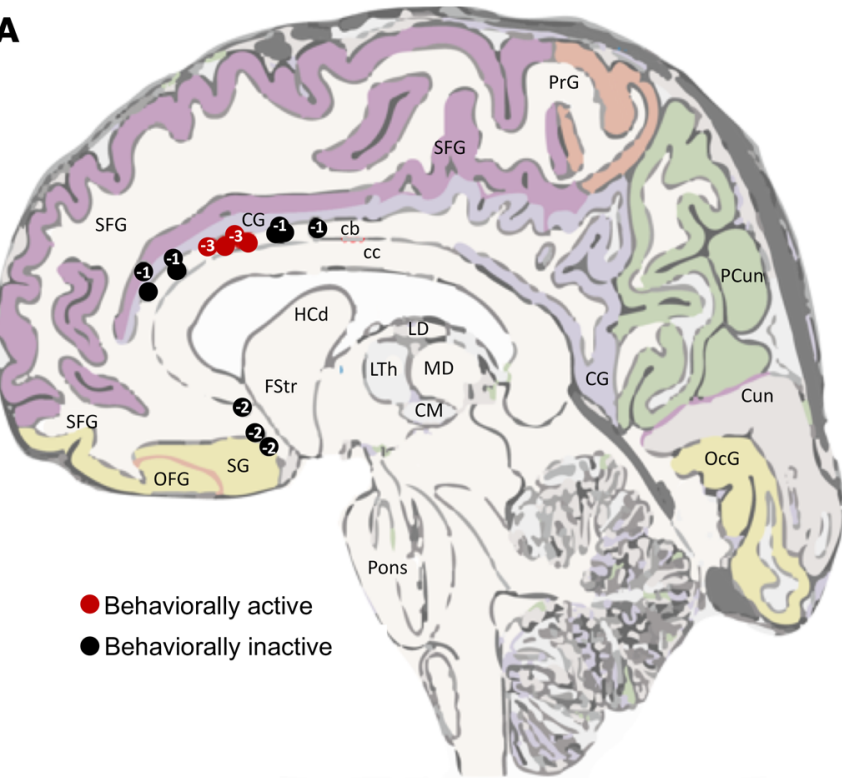

B

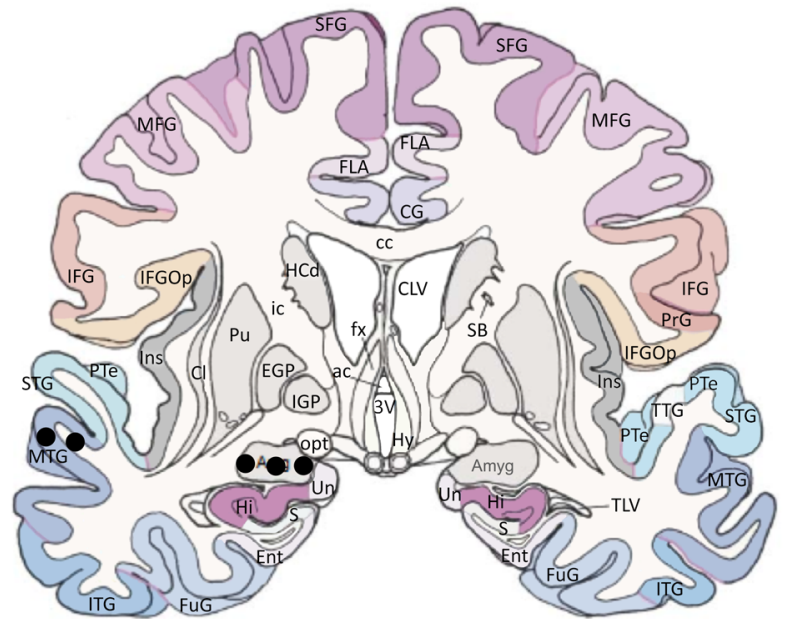

component of her typical seizure or aura. She reported an involuntary urge to laugh that began at the onset of stimulation and evolved into a pleasant, relaxed feeling over the course of a few seconds of stimulation. Following the offset of stimulation, the sensation dissipated over the course of a few seconds. Videographic recordings showed that the smile sequence began with contraction of the right zygomaticus muscle before spreading across the face into a natural-appearing smile (Supplemental Videos 1-3).

Progressively increasing stimulation amplitudes from 1.5 to $3.5 \mathrm{~mA}$ produced subjectively similar but more intense experiences, while single-blinded sham stimulations elicited no such subjective or objective changes. At 3.0 and $3.5 \mathrm{~mA}$, the patient experienced even more pronounced and sustained laughter and mirth, stating, "Wow, everyone should have this," and that she was "so happy she could cry." The urge to laugh remained time-locked with stimulation onset and lasted longer at greater stimulation amplitudes, but resolved over several seconds of continuous stimulation into her feeling "contented," "happy," and "relaxed." Similar effects of stimulation to this contact pair were observed at a lower frequency with higher current settings $(50 \mathrm{~Hz}, 200 \mu$ s, 1.5-3.5 mA, data not shown).

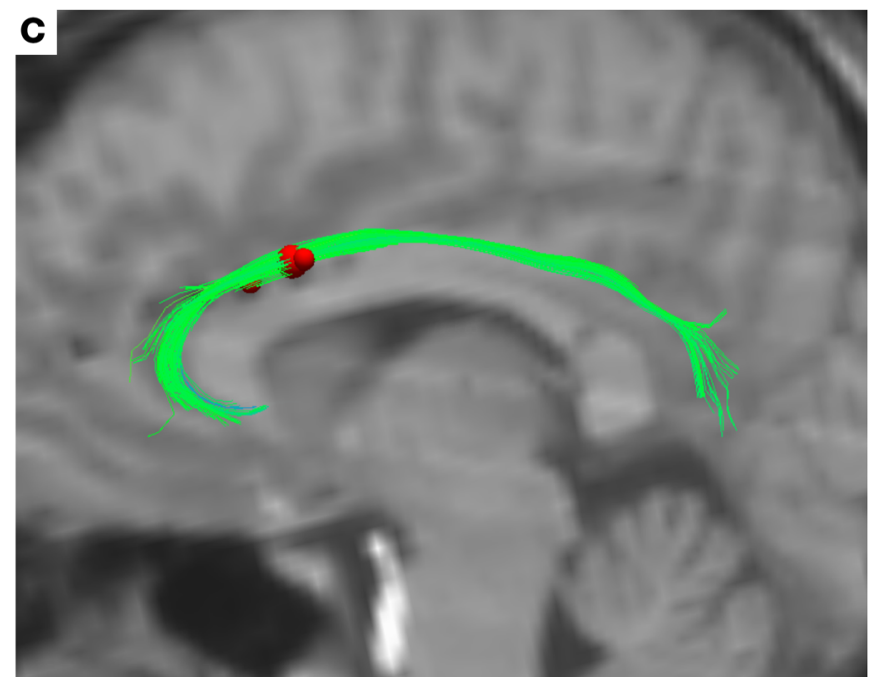

Figure 2. Localization of stimulated electrodes for the $\mathbf{3}$ patients. (A and B) Sagittal and coronal views of stimulated locations across the full patient set $(n=3)$. Numbers inside circular indicators on the sagittal view reflect the number of 1-mm incremental sections from the displayed section in which the contact was located, where "-" corresponds to sections more medial and "+" corresponds to sections more lateral. Red circles indicate behaviorally active contacts, whereas black circles indicate behaviorally inactive contacts. (C) Overlap of the volumes of tissue activated (red) by all behaviorally active contacts across the full patient set $(n=3)$, relative to the index patient's diffusion tensor imaging data set, collapsed by side of stimulation. The VTA for all patients robustly engage the cingulum bundle to the exclusion of other fiber systems. cb, cingulum bundle; CG, cingulate gyrus; PrG, precentral gyrus; PCun, precuneus; SFG, superior frontal gyrus; Cun, cuneus; OcG, occipital gyrus; cc, corpus callosum; OFG, orbitofrontal gyrus; SC, straight gyrus; HCd, head of caudate nucleus; FStr, fundus striati; LTh, lateral thalamic nucleus; MD, mediodorsal thalamic nucleus; CM, centromedial thalamic nucleus; LD, lateral dorsal thalamic nucleus; MTC, middle temporal gyrus. The underlying anatomical drawings in $\mathbf{A}$ and $\mathbf{B}$ were adapted with permission from Elsevier (52).

Next, we examined the effect of stimulating the adjacent, more posterior contact pair within the cingulum bundle underlying the mid-cingulate cortex $(130 \mathrm{~Hz}, 300 \mu \mathrm{s})$ (Figure 1). At $1.5 \mathrm{~mA}$, the patient reported a "high" sensation, without any facial sensation or observed smiling or laughter. At $2.0 \mathrm{~mA}$, the patient reported that the stimulation felt to be "too much" and described a pulling sensation of her head toward the right, different from prior stimulations and less pleasant. Thus, stimulation at this more posterior cingulum contact pair was discontinued (Supplemental Table 1).

Behavioral replication. Stimulation of the right cingulum bundle was examined in 2 additional patients with similar electrode array placements (anatomical details are presented in Figure 2 and Supplemental Figures 1 and 2; trial-by-trial transcription of stimulation testing is provided in Supplemental Tables 2 and 3). In each subsequent case, stimulation of the contact pair most analogous to that of the index patient's effective contacts yielded the strongest behavioral responses.

In patient 2, bipolar stimulation in the right cingulum bundle was delivered over 2 pairings of 3 contiguous contacts, mirroring those in the index patient (Figure 2) at very similar stimulation parameters. In the anterior contact pair, smiling was elicited at 2.5 


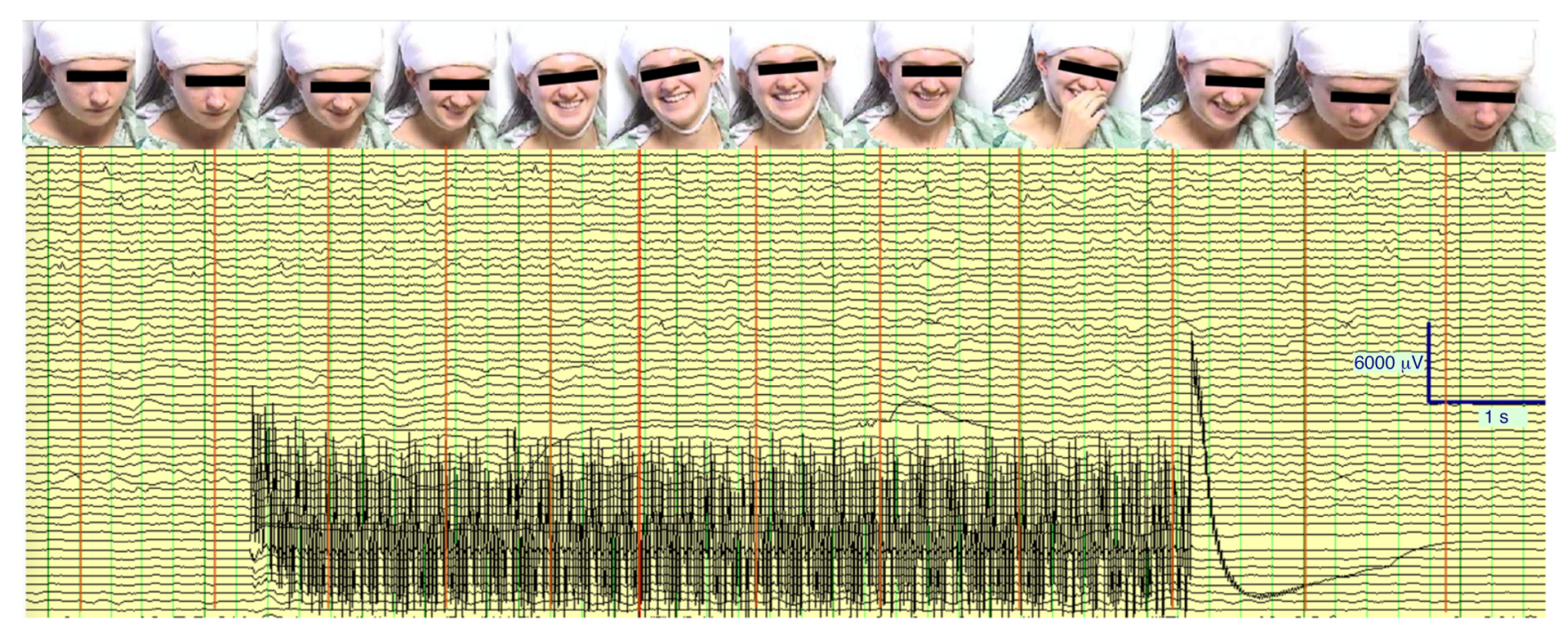

Figure 3. Mirth induced by acute cingulum stimulation in the index patient. Continuous intracranial EEG record and associated sequential still images of patient's face showing temporal progression of facial expression (smiling, laughter) during a 6 -second period of acute cingulum bundle stimulation (3.0 $\mathrm{mA}, 130 \mathrm{~Hz}, 300-\mu \mathrm{s}$ pulse width marked by stimulation artifact). No epileptiform after-discharges from cingulum stimulation were detected in any channel. The patient described the experience as follows: "I just feel my face smile and I feel happy. [The happiness] lingers for a while and then it kind of slows down a little and I just feel happy and relaxed."

$\mathrm{mA}(130 \mathrm{~Hz}, 300-\mu \mathrm{s}$ pulse width, 5 -s duration), mood elevation was reported at $3.0 \mathrm{~mA}$, and overt laughter occurred at $3.5 \mathrm{~mA}$. Interleaved sham stimulations produced no behavioral or subjective effects. Stimulation of the more posterior contact pair, in addition to smiling at $2.0 \mathrm{~mA}$, also elicited an urge to move at $2.5 \mathrm{~mA}$. By comparison, control stimulation to a nonlimbic structure (the middle temporal gyrus) caused no subjective or overt behavioral changes.

In patient 3, we used a portable neural stimulator (DualStim 3628, Medtronic), and ratings of happiness, relaxation, and pain were given using visual analog scales (scale: 0-10) for each. At baseline, the patient rated her happiness as 8 out of 10, her relaxation as 4 out of 10, and her postoperative scalp pain was 4 out of 10. Compared with other contact pairings, the pair most analogous to that placed in the index patient's contralateral cingulum bundle, when stimulated at $3 \mathrm{~V}(130 \mathrm{~Hz}, 300$ - $\mu$ s pulse width, 5 -s duration), yielded smiling and laughter, a 10\% increase in happiness, a $20 \%$ increase in relaxation, and a $20 \%$ reduction in pain. Stimulation at $5 \mathrm{~V}$ elicited similar behavior and, compared with baseline, a $20 \%$ increase in happiness, a $60 \%$ increase in relaxation, and a $40 \%$ reduction in pain. Patient-blinded comparison with sham yielded a rapid return to baseline pain. Longer 3-minute stimulation periods at $3 \mathrm{~V}$ and $4 \mathrm{~V}$ provided continuous mood elevation, self-professions of optimism, and continuous pain relief. At $4 \mathrm{~V}$, the patient was asked to describe a sad memory, during which she was observed to be smiling, and she described not feeling sad despite accurate recollection. Baseline levels of pain again recurred when stimulation was discontinued.

\section{Cingulum stimulation maintained positive affect without other changes in mental status}

To ascertain the effects of more prolonged anterior cingulum bundle stimulation and characterize any impact on autonomic parameters and mental status in the index patient, unblinded stimula- tion was delivered continuously at $2 \mathrm{~mA}, 130 \mathrm{~Hz}$, and $300-\mu$ s pulse width for 33 minutes. These parameters were selected because they had been previously shown to produce consistent behavioral changes in the patient and were tolerable with regard to intensity (see Stimulation in Methods for details on amplitude selection). No electrographic or clinical seizures were observed during this period, nor were subjective auras reported.

During continuous stimulation, the patient was evaluated using a standard mental status examination (21) and recorded for later transcription and assessment by an independent rater (see Supplemental Table 1 and Supplemental Videos 1-3). Attitude changed from the onset of stimulation from slight disengagement to continuous engagement with increased rapport. Behavior was notable for increased eye contact without overt psychomotor activation. Mood shifted from reports of anxiety to initial euphoria, then happiness and relaxation. Congruently, affect changed from flat to reactive, with smiling, joking, and socially appropriate laughter. Speech grew more spontaneous and productive, without being pressured or rapid. Thought process was normal in quantity, tempo, coherence, and linearity. Thought content, although preoccupied with the pending surgery, was without delusion, obsession, phobias, or negative cognitive distortions. Although perception was altered by the external influence of cingulum stimulation, the patient reported the perceived involuntary nature of her facial expression and laughter to be pleasant rather than disturbing. She reported being unable to voluntarily produce a frown without laughing. She denied hallucinations and auras. Her cognition showed alertness, and she recalled long-term memories without difficulty. When asked to think of a sad memory, she accessed the semantic content of a memory, stating, "I remember my dog dying, and I remember that it was a sad memory, but I don't feel sad about it right now." The patient's descriptions during stimulation exhibited intact insight. Judgment was not formally test- 
Table 1. Summary of bedside preresection observations during sham and active stimulation of the left dorsal cingulum bundle (contacts 3-4 in Figure 1)

\begin{tabular}{|c|c|c|}
\hline Trials & Current $(\mathrm{mA})$ for $5 \mathrm{~s}$ & Patient self-report and research observations \\
\hline $1-3$ & $0.5 \mathrm{~mA}$ & Denies subjective experience. \\
\hline $4-7$ & $1.0 \mathrm{~mA}$ & Smiles and chuckles involuntarily: reports "feeling something", smiling because she "can't help it." \\
\hline $8-10$ & $1.5 \mathrm{~mA}$ & $\begin{array}{l}\text { Smiles, feels forehead "twitch" (pulling upward) and feels smile more on her right, like she is "not in control," as might happen during a } \\
\text { seizure, but denies this is her typical semiology. Feels "restless" and a change in mood. "Just a feeling of happiness". }\end{array}$ \\
\hline $11-12$ & $2.0 \mathrm{~mA}$ & Smiles and laughs. "It felt the same, just more intense, in a good way...That's awesome!". \\
\hline 13 & $2.5 \mathrm{~mA}$ & Feels happy, more eyebrow "twitching" (pulling upward). \\
\hline 14 & $0 \mathrm{~mA}$ (sham) & Denies subjective experience. \\
\hline 15 & $3.0 \mathrm{~mA}$ & Smiles and laughs. Feels happy and a right-sided smile. \\
\hline $16-17$ & $3.5 \mathrm{~mA}$ & Smiles and laughs. Feeling is more intense: "Wow, everyone should have this... I'm so happy I want to cry." \\
\hline
\end{tabular}

5-second stimulation trials $(130 \mathrm{~Hz}, 300-\mu$ s pulse width, 10 - to 60 -s interstimulation intervals for interview and verbal responses). The patient's self-report was aggregated by an independent rater who reviewed the videos of all patient testing, transcribed all responses, and synthesized summary tables and summary statements. For trial-by-trial details on all stimulation testing, see Supplemental Table 1.

ed, but there was no evidence of changes with respect to making sound, reasoned, and responsible decisions. The day after stimulation, the patient reported that her sleep was unusually restful and that she felt optimistic about surgery.

In patient 2, we formally assessed neuropsychological functioning during continuous stimulation to the cingulum bundle over a period of 64 minutes with concurrent electrophysiological monitoring (Supplemental Figure 1 indicates stimulated contacts and cingulum bundle engagement). No electrographic or clinical seizures were observed during this period, nor were subjective auras reported. Stimulation was delivered continuously at a frequency of $40 \mathrm{~Hz}$, using a 300- $\mu$ s pulse width and $3.2 \mathrm{~V}$ (as titrated to be just below the patient's subjective threshold for evoked smiling at $3.5 \mathrm{~V}$, which the subject reported to find potentially “distracting"). The patient's delayed verbal recall was observed to be $1.5 \mathrm{SD}$ worse during stimulation than prior to surgery on a single, complex list-learning task (Rey Auditory Verbal Learning Test [RAVLT]). His delayed recall was 10 of 15 when not stimulated and 6 of 15 when stimulated. However, there was no detectable disturbance across multiple measures of attention, processing speed, immediate recall of information or any tested language, or executive function performances. Thus, overall, the neuropsychological impact of stimulation in this patient appeared minimal. Additional neuropsychological details are provided in Supplemental Table 4.

\section{Cingulum stimulation drove a positive shift in affective bias and facial expression dynamics}

To obtain objective correlates of mood, the index patient completed a facial affective bias task, in which static images of emotional facial expressions are presented and the patient rates the intensity and valence of each expression (22-24). The patient completed the task during six 10-minute blocks comparing multiple conditions: (a) sham stimulation; (b) left dorsal anterior cingulate cortex (gray matter) at the highest level of stimulation previously tested and which did not alter subjective experience $(3.5 \mathrm{~mA})$; (c) left dorsal anterior cingulum (white matter) at an amplitude just below subjective experience $(0.5 \mathrm{~mA})$; and (d) left dorsal anterior cingulum (white matter) at the lowest amplitude that produced a subjective experience (1.0 mA). As demonstrated in Supplemental
Figure 3, compared with sham, maximal stimulation of cingulate cortex gray matter and subthreshold cingulum white matter stimulation yielded modest positive shifts in affective bias. By contrast, suprathreshold stimulation of cingulum produced typical initial mirth and a strong positive shift in the ratings of emotional faces, in which all faces were interpreted as happier during stimulation than during sham (Supplemental Figure 3).

As a second objective metric of stimulation effects, we examined video records of the patient's face via an unsupervised machine-learning algorithm (25) that was able to discriminate dynamic differences in patterns of intensity change by pixel in the patient's facial features over time with each experimental condition. This analysis independently showed distinct facial motor patterns associated with sham, subthreshold, and suprathreshold stimulation (Supplemental Figure 8), providing a potential biomarker of cingulum stimulation beyond self-reporting of a change in emotion.

As a third objective measure of stimulation effects, we analyzed autonomic responses in terms of skin conductance response and heart rate across 30-second epochs of stimulation, in which we observed a dose-response relationship with greater skin conductance and heart rate observed with increasing levels of stimulation amplitude, as presented in Supplemental Figure 4. Behavioral observations of this testing are presented in Table 2. The patient's heart rate was clinically recorded as the mean \pm standard error (SE): $79.3 \pm 3.0 \mathrm{bpm}$ over a 7-day period inclusive of stimulation studies. During continuous monitoring, the 30-minute prestimulation baseline was recorded as: 101.52 mean $\pm 2.35 \mathrm{SE}$ (range 85-130 bpm). With continuous stimulation, the heart rate monitoring was intermittently confounded by frequent laughter episodes and movement, but yielding a slightly broader dynamic range of: 113.31 mean $\pm 3.53 \mathrm{SE}$ (range $83-160 \mathrm{bpm}$ ).

\section{Changes in intracranial local field potentials were associated with cingulum stimulation}

We measured changes in local field potentials (LFPs) as a means to assess the impact of stimulation upon subsequent brain activity and network interactions with the cingulum bundle. Twenty replications of transient (1-s) stimulation were delivered at $130 \mathrm{~Hz}$ 


\section{Table 2. Summary of bedside preresection observations during sham and active $\mathbf{3 0 - s e c o n d ~ s t i m u l a t i o n ~ o f ~ t h e ~ l e f t ~ d o r s a l ~ c i n g u l u m ~}$ bundle (contacts 3-4 in Figure 1) with recorded psychophysiology}

\begin{tabular}{|c|c|c|c|}
\hline Trial & Current (mA) & EDA & Patient self-report and research observations \\
\hline 1 & 0 mA (sham) & + & Denies subjective experience. \\
\hline 2 & $0.5 \mathrm{~mA}$ & + & Denies subjective experience. \\
\hline 3 & $1 \mathrm{~mA}$ & ++++ & $\begin{array}{l}\text { Smiles and laughs. Feels smiling on the right side and feels happy; this is accompanied by a large, initially positive EDA (+ EDA) } \\
\text { response at stimulation onset, with slow trend back toward baseline. }\end{array}$ \\
\hline 4 & 0 mA (sham) & - & No smile or laugh, but reports persistent relaxation and happiness. \\
\hline 5 & $1.5 \mathrm{~mA}$ & +++ & Smiles and laughs. Stronger feeling. Could feel it dissipate after the offset of stimulation. \\
\hline 6 & $2 \mathrm{~mA}$ & +++ & More persistent laughter. Feels more persistently relaxed. \\
\hline 7 & $0 \mathrm{~mA}$ (sham) & - & Denies subjective experience. \\
\hline 8 & $0 \mathrm{~mA}$ (sham) & + & Denies subjective experience. No longer feels relaxed; a spontaneous thought about pending surgery provokes anxiety. \\
\hline 9 & $2.5 \mathrm{~mA}$ & +++ & "Happy feeling" persisting past the offset of stimulation. \\
\hline 10 & $3.0 \mathrm{~mA}$ & ++ & Stronger sensation, lasting longer. Feeling relaxed; denies anxiety. \\
\hline 11 & $3.5 \mathrm{~mA}$ & +++ & Same feeling as just before; feels involuntary right-sided smile. \\
\hline 12 & 0 mA (sham) & - & Denies subjective experience; neither relaxed nor anxious; no facial motor feeling. \\
\hline
\end{tabular}

30 -second stimulation trials with autonomic physiology recording $(130 \mathrm{~Hz}, 300-\mu$ s pulse width, $30-$ to $60-\mathrm{s}$ interstimulus interval, verbal response, and recovery of autonomic baseline). Autonomic recordings are shown in Supplemental Figure 4. Patient self-report was aggregated by an independent rater who reviewed the videos of all patient testing, transcribed all responses, synthesized summary tables and summary statements. The patient showed a mild (1.5 microSiemens $[\mu \mathrm{S}]$ ) EDA response with the first sham stimulation, consistent with experimental anticipation. All other sham stimulations elicited EDA responses below $1.0 \mu \mathrm{S}$, with the exception of trial 8 , in which the patient reported a spontaneous thought about pending surgery and reported feeling anxious about it. In the EDA column, "-" indicates a change of less than $1.0 \mu \mathrm{S}$, "+" indicates change of 1.0 to $2.9 \mu \mathrm{S}$, "++" indicates change of 3.0 to $5.9 \mu \mathrm{S}$, "+++" indicates change of 6.0 to $9.9 \mu \mathrm{S}$, and "++++" indicates a change of more than $10.0 \mu \mathrm{S}$. For details on autonomic analysis, see Supplemental Figure 1 . For trial-by-trial details on all stimulation testing, see Supplemental Table 1.

and $3.5 \mathrm{~mA}$, with 10 interleaved sham stimulations (single-blind false stimulation condition), delivered at 10 -second interstimulus intervals, during the recording of LFP data from 127 of the patient's implanted subdural and stereotactic depth electrode contacts sampling medial and lateral aspects of the left frontal and parietal lobes and medial, lateral, and basal aspects of the bilateral temporal lobes, the medial and lateral aspects of the parietal lobes, and the right amygdala (Figure 4, Supplemental Figure 5, Supplemental Figure 6, and Supplemental Video 3). Cingulum stimulation consistently and immediately elicited a brief smile, while there were no observable reactions to sham stimulation. For comparison with natural social smiling behavior, we also collated 10 episodes of spontaneous social smiling from previous days' videographic EEG recordings unrelated to stimulation.

Power spectral density was examined on all recorded channels between 0 and $100 \mathrm{~Hz}$ (Supplemental Figure 7), and LFPs in the 6- to $11-\mathrm{Hz}$ range (spanning frequency ranges that are frequently labeled in humans as alpha and theta ranges) were found to be prominent across all electrode contact locations. The peak frequency was often narrower (approximately 7 to $8 \mathrm{~Hz}$ for many electrode locations), but analyses used the broader $6-$ to $11-\mathrm{Hz}$ range to encompass the observed range of activity. LFPs at 6 to $11 \mathrm{~Hz}$ were analyzed for changes in power at each recorded electrode location and for changes in coherence between each location and the cingulum bundle following offset of cingulum stimulation relative to a prestimulation baseline period (see Method for details). Specifically, power and coherence averaged across 3 seconds prior to the onset of stimulation ( $-4 \mathrm{~s}$ to $-1 \mathrm{~s}$ prior to onset) were subtracted from power and coherence averaged across $3 \mathrm{sec}-$ onds following the offset of stimulation $(+1 \mathrm{~s}$ to $+4 \mathrm{~s}$ after offset).
The 1-second period of stimulation (plus a 1-s window before and after) was excluded because of stimulation artifacts.

To evaluate separately for each electrode contact whether the poststimulation changes in power and coherence differed from random chance, we evaluated the statistical significance of the 6to $11-\mathrm{Hz}$ range across all recorded electrode contact locations relative to sham stimulation (Figure 4). From the location of the cingulum bundle electrode contacts, we recorded a persistence of the endogenous 6- to 11-Hz LFPs following sham stimulation, whereas active stimulation was followed by a strong reduction in $6-$ to $11-\mathrm{Hz}$ power (Figure 4A). Following stimulation, 6- to $11-\mathrm{Hz}$ power and coherence were reduced broadly over the left hemisphere (including motor and sensory areas of the face), but power was increased in the medial temporal lobes (Figure 4B). A random permutation approach (see Methods) was used to evaluate, for each electrode contact, whether the poststimulation difference in 6- to $11-\mathrm{Hz}$ power and coherence relative to baseline was unlikely $(P<0.05)$ to be due to chance. The overall network effect was evaluated using basic probability (binomial probability modeled using the "binopdf" function in MATLAB 2017a, MathWorks). For power, 43 versus 7 of 127 electrodes showed significant changes following stimulation versus sham, respectively. If the random probability of an individual significant test is 0.05 , the probability of observing 43 of 127 or more significant tests is not likely to be due to random chance $(P<0.001)$. By comparison, the probability of observing 7 of 127 significant tests would be $P=0.451$. For coherence with contacts in the cingulum bundle, 27 of 126 contacts showed significant changes following stimulation $(P<0.001$; Figure $4 C)$, whereas sham stimulation produced significant changes in 2 of 126 electrodes $(P=0.988)$. Thus, the number of channels showing statistically significant changes in 
A 6 - to $11-\mathrm{Hz}$ power reduced with

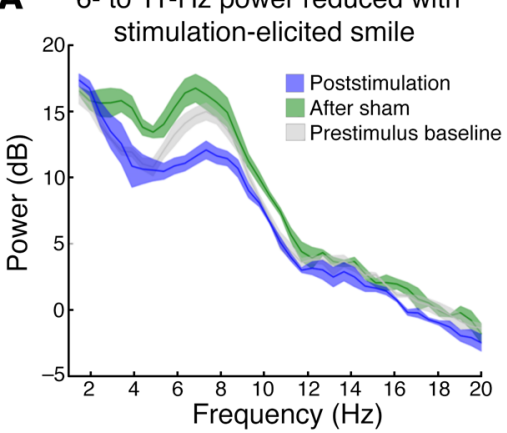

6- to $11-\mathrm{Hz}$ power reduced with

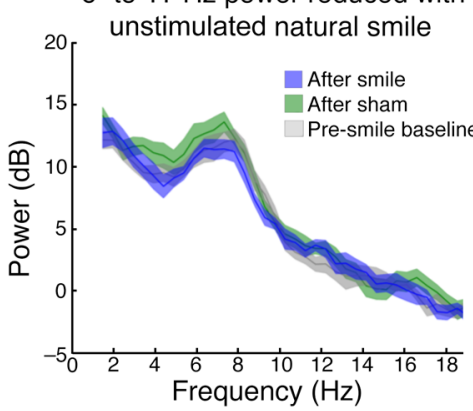

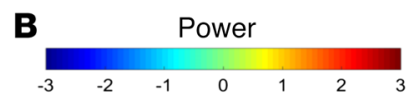
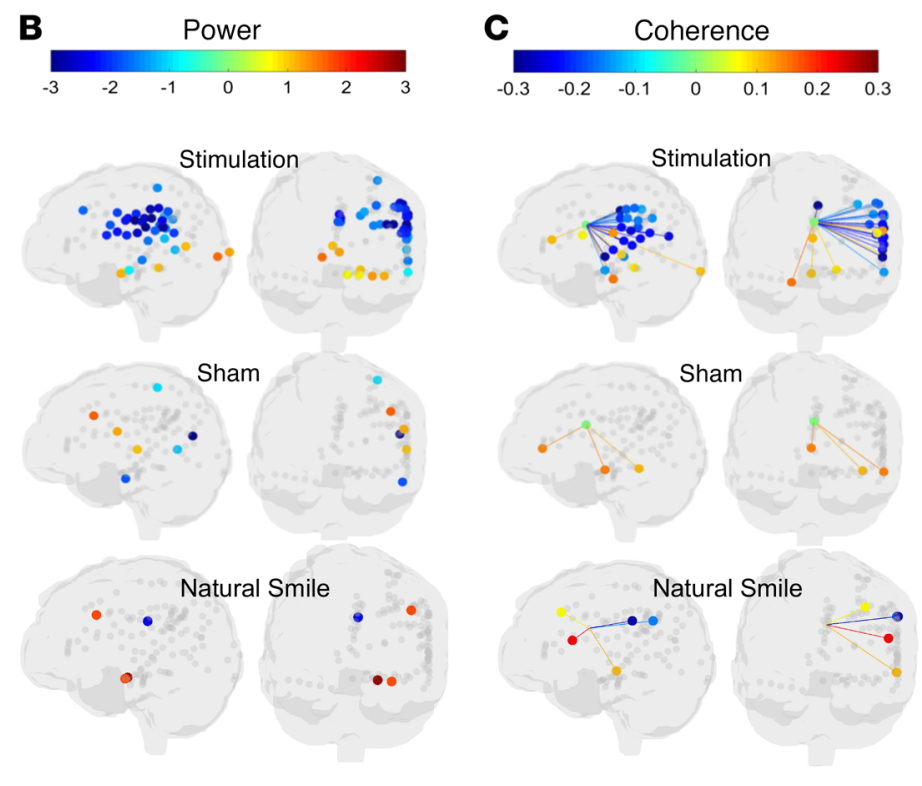

Figure 4. Distinct neural activity patterns associated with stimulation-induced mirth in the index patient. (A) Top: LFP shows reduced power in decibels (dB) of an endogenous cingulum oscillation of 6 to $11 \mathrm{~Hz}$ following stimulation (blue) compared with prestimulus baseline (gray), but not following nonstimulated sham (green). Bottom: Ten natural (unstimulated) social smiles were analyzed during intracranial recording. The endogenous oscillation of 6 to $11 \mathrm{~Hz}$ was again observed in the naturalistic data set at the pre-smile baseline (gray) and following sham LFP epochs in which smiling was not present (green) and showed no evidence of reduced power associated with natural smiling (blue). Lighter-colored areas indicate $95 \% \mathrm{Cls}$ generated by a random reshuffling procedure. (B) LFP shows distinct reduction in power across the recorded lateral-opercular frontal-parietal lobe network (including ipsilateral motor and sensory cortices of the head and face) following stimulation, but not following sham stimulation or unstimulated natural smiles. Ventral ACC, amygdala, parahippocampus, and medial precuneus showed increased in 6- to 11-Hz power (increased synchrony) following cingulum stimulation. (C) After stimulation, LFP likewise showed reduced network coherence between the cingulum and multiple contacts corresponding to the ipsilateral motor/sensory cortices of the head/face, but not following sham stimulation or unstimulated natural smiles. For $\mathbf{B}$ and $\mathbf{C}$, the colored dots indicate electrode locations and the corresponding power, using the heatmap as indicated in the legend. Only contacts with statistically significant changes in power or coherence are presented with colored dots; gray dots indicate contact locations with nonsignificant changes in power or coherence. Statistical significance reflects the percentile of the null (random) distribution at which the actual value was observed. For more precise colocalization of significant contacts within the anatomic MRI, see Supplemental Figures 2 and 3.

6- to $11-\mathrm{Hz}$ power (43 of 127$)$ and coherence (27 of 126$)$ following stimulation was very unlikely to have occurred by chance.

To explore whether the observed changes in the pattern of LFPs resulted directly from cingulum stimulation versus the natural physical act of smiling, episodes of spontaneous (unstimulated) social smiling were compared with pre-smile baselines using the same method. By contrast to stimulation-elicited mirth, spontaneous social mirth exhibited no appreciable decrease in 6- to 11-Hz power relative to pre-smile baseline, nor did we observe any robust pattern of shifts in power or coherence across the sampled networks (Figure 4). While we did not control the electrophysiological state just prior to spontaneous social mirth versus stimulation-elicited mirth, the rapid dynamic shift induced by cingulum stimulation is unlikely to be a pure electrophysiological correlate of the physical act of smiling.

Part II: Clinical translation: cingulum stimulation repeatedly suppressed anxiety without sedation during awake brain surgery Spontaneous seizures were localized via implanted electrodes to the left posterior temporal lobe just posterior to the basal temporal language area. Standard clinical language disruption mapping at $50 \mathrm{~Hz}$ identified an overlapping region of eloquent cortex. Thus, an awake procedure with continuous language testing was proposed to protect eloquent cortex during resection. The patient expressed significant anxiety at the prospect of awake surgery, and her baseline BAI had suggested a clinically significant symptom burden. We proposed that cingulum stimulation to elicit intraoperative anxiolysis would facilitate the procedure. A few hours prior to surgery, mirth and positive affect were again observed from cingulum stimulation, this time using a portable neural stimulator (DualStim 3628, Medtronic) connected to the intracranial lead, at a threshold of $2 \mathrm{~V}, 130 \mathrm{~Hz}$, and $300 \mu$ s of stimulation.

The patient underwent a standard surgical sequence including cranial opening under deep sedation and then awake resection, followed by resumption of sedation for closure (Figure 5). Total intravenous anesthesia with short-acting agents (propofol and dexmedetomidine) was maintained, and a laryngeal mask airway supported artificial respiration. She underwent circumferential scalp blockade anesthesia. The patient was oriented in a semilateral position to maximize surgical exposure of the left temporal 


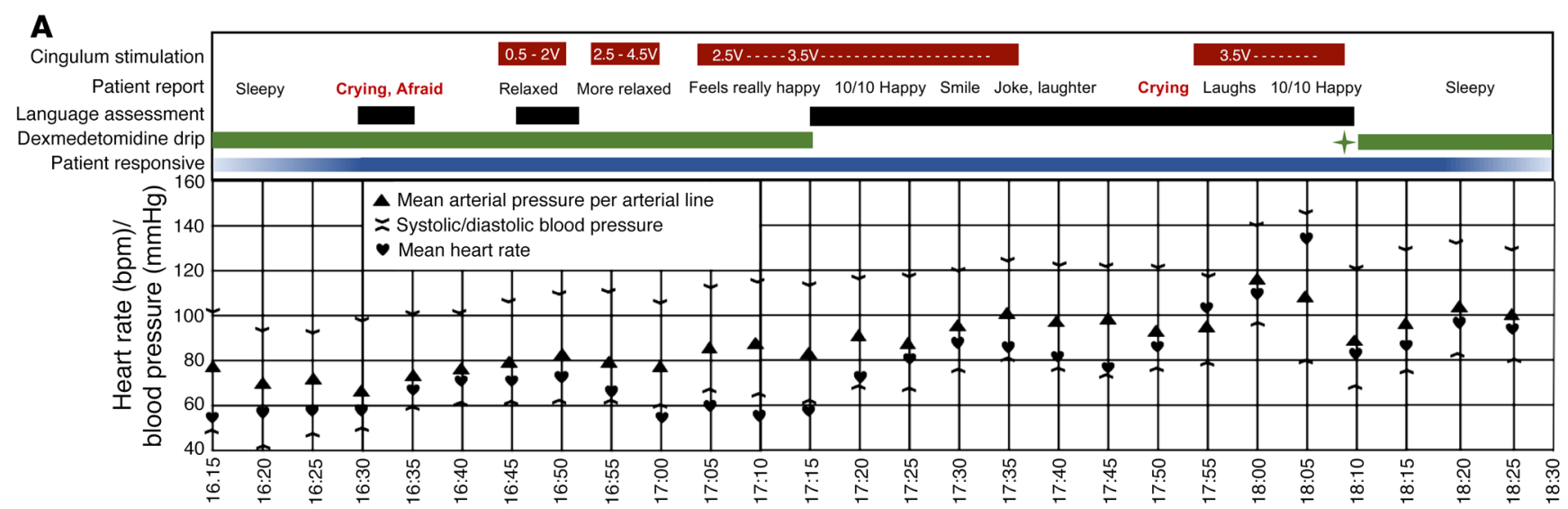

Time (hours:minutes)

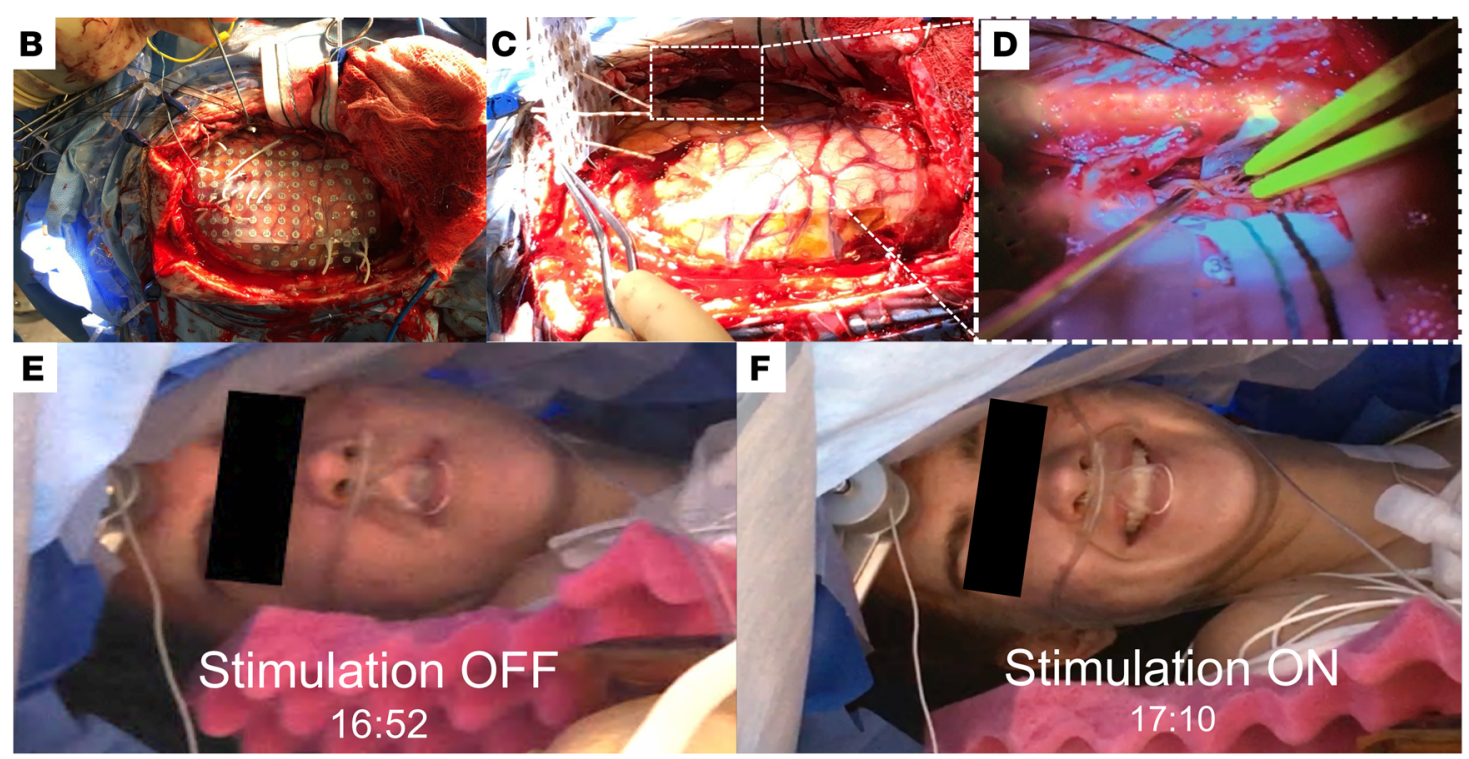

Figure 5. Intraoperative timeline, photographs, and impact of stimulation on tolerance of awake brain surgery in the index patient. (A) Chart shows the timing of the intraoperative interventions, and observations are indicated in the color bars at the top; periodic measurements of heart rate and blood pressure are reported in the lower grid. Initially, continued dexmedetomidine (a sedative-anxiolytic agent) infusion alone was associated with periods of both sedation and anxiety, which confounded the initial language assessment. As cingulum stimulation was titrated up to maintain anxiolysis, dexmedetomidine was ultimately discontinued completely, and language assessments were performed successfully. For a period during awake surgery, cingulum stimulation was discontinued as well, resulting in recurrent anxiety, but this was again immediately alleviated by resuming stimulation. After the completion of the language assessment, a bolus of propofol (sedative anesthetic) was administered (green star), dexmedetomidine was reinitiated, and cingulum stimulation was discontinued. All intraoperative stimulation was voltage-controlled at $130-\mathrm{Hz}$ frequency, $300-\mu$ s pulse width and was delivered in a bipolar, biphasic, and charge-balanced manner. Blood oxygenation remained at $100 \%$, and other vital signs were stable throughout the procedure. (B-F) Intraoperative images show the spatial extent of the grid coverage with 1-cm-spaced electrode contacts over the left lateral surface of the brain (B), the appearance of the brain after lifting off a grid array with depth electrodes, with an inset (dotted white rectangle) focused on the location of the resection (C), and a magnified view of the location of the resection in the posterior inferior and middle temporal gyri (D). For size reference in B, electrocorticography grid contacts are spaced at $1-\mathrm{cm}$ intervals, and for size reference in $\mathbf{D}$, the white surgical cotton strip pointing into the surgical field is 0.5 in. wide. The patient is positioned laterally, with the head parallel to the floor and left side up, creating a space to examine her beneath the surgical drape. Intraoperative images of her face without stimulation (E) and with anxiolytic stimulation (F), showing a characteristic smile.

region, with the face exposed for neurological evaluation (Figure 5). The cranial flap was removed, the dura reopened, and the underlying subdural electrodes and brain exposed.

Maintenance intravenous anesthesia was titrated down, spontaneous breathing resumed, the laryngeal airway was removed, and the patient became aware and responsive over the course of several minutes, upon which she became tearful and reported significant anxiety and fear, but denied pain. The surgeon and neurologist provided verbal reassurance, and cingulum stimulation was offered for anxiolysis. Stimulation was initiated at $0.5 \mathrm{~V}$ while monitoring electrocorticography, titrating to $4.5 \mathrm{~V}$ without eliciting epileptiform after-discharges. Beginning at $1.5 \mathrm{~V}$, and increasing in intensity with greater amplitudes of stimulation, the patient reported feeling more relaxed and began to smile, laugh, and joke. She recalled crying with fear upon becoming aware and reported feeling comparatively at ease during stimulation. When 
stimulation was briefly discontinued, the patient reported feeling more pain in her scalp, became aware of the use of electrocautery, and expressed anxiety. Additional lidocaine was percutaneously injected at the scalp around the incision, and stimulation was reinitiated to $3.5 \mathrm{~V}$, after which the patient rapidly reported feeling "really happy," rated her happiness as a 10 out of 10 (10 being the happiest she had ever felt), and denied pain. She reported residual drowsiness, so her remaining infusion of short-acting sedative/ analgesic (dexmedetomidine) was completely discontinued, and cingulum stimulation was continued in the absence of any general anesthetic agent. Thereafter, stimulation was applied at $2.5 \mathrm{~V}$ and later elevated to $3.5 \mathrm{~V}$ during the language testing, without a general anesthetic agent. Upon initiation of the language testing (naming objects, animals, and famous faces; reading and answering written questions; participating in unstructured conversation), her object, animal, and famous faces naming was determined to be primarily intact, and she maintained the ability to read and answer written questions correctly. She participated as needed in language testing throughout the temporal cortical resection. Dexmedetomidine sedation was only resumed following completion of the language assessment and resection, almost an hour after previous discontinuation.

Notably, during the language-mapping task, cingulum stimulation was inadvertently discontinued for a period of time without being recognized by the patient or the clinical team, providing an unintended intraoperative double-blind, controlled experiment (Figure 5). The patient became unexpectedly dysphoric (acutely tearful and reported increasing anxiety and fear), resulting in temporary suspension of language testing and surgical resection. The absence of prior stimulation artifact in electrocorticography was identified. Upon resumption of cingulum stimulation, however, her anxiety immediately abated, and shortly thereafter she again rated her happiness at 10 out of 10 . While there was a recognizable upward trend in intra-arterial blood pressure and heart rate following unintended discontinuation of stimulation, her blood pressure remained in an expected range (systolic range $=118-142$ $\mathrm{mmHg}$, diastolic range $=60-95 \mathrm{mmHg}$ ), her heart rate remained between 58 and $136 \mathrm{bpm}$, and her blood oxygenation remained at $100 \%$ throughout the awake portion of the surgery (Figure 5). A retrospective review of the electrocorticographic records pinpointed the offset of stimulation approximately 17 minutes prior to dysphoria, suggesting a washout period following continuous cingulum stimulation. After resuming stimulation, language testing and resection were continued.

Over the course of the resection, the patient eventually began making language-related errors in naming famous faces and specific types of animals, which prompted the decision to discontinue further resection. Intravenous sedation was resumed, cingulum stimulation was discontinued, and the remaining surgery was completed. Her remaining postoperative course was uncomplicated, and she later recalled the awake portion of her surgery as a positive experience.

The index patient continued to be nondepressed 6 months and 12 months after her surgery (BDI-II $=2$ at the 6-month postoperative time point; $\mathrm{BDI}-\mathrm{II}=3$ at the 12 -month postoperative time point), and her postoperative anxiety measures improved (from $\mathrm{BAI}=22$ preoperatively to $\mathrm{BAI}=10$ at both the 6 -month and 12-month time points), suggesting a persistent postoperative reduction in anxiety that was probably not related to stimulation.

\section{Discussion}

Technical considerations. A technical advantage of our study is the high density and favorable orientation of electrode contacts along a novel trajectory through the cingulum bundle. Typically, clinical depth electrodes sample small cross sections of cingulate cortices from an orthogonal (lateral to medial) approach, in which any single electrode array is likely to intersect the cingulate region perpendicularly with only 1 to 2 contacts, and numerous arrays would be required to sample the extent of the cingulate cortices. In our case, because of a clinical hypothesis regarding seizure onset, we designed a high density of electrode contacts sampling cingulate regions. The surgeon used novel stereotactic trajectories aligned to the long axis of sections of the cingulum bundle, yielding a uniquely broad capacity for recording and stimulation of the cingulate regions in a single patient. Alignment of trajectories with the cingulum bundle also resulted in placement of multiple contiguous contact pairs wholly within white matter, yielding dipoles and stimulation fields parallel to axonal projections. This configuration is more likely to activate axons than when exposed to orthogonal dipoles (26). Relative to gray matter, stimulation of white matter provides more direct connectivity to near and distant structures and generates complex orthodromic and antidromic effects (27). Thus, the observed sensitivity of behavioral responses at low stimulation amplitudes may have resulted from the unique alignment of contact pairs within the cingulum bundle. To our knowledge, such a concentrated study of stimulation along the dorsal cingulum bundle has never been described. While electrical brain stimulation is a therapeutic modality and the gold standard for anatomical-functional localization in human brain, more precise cellular functional localization with molecular and optogenetic techniques in experimental animals may help to causally link behavioral and physiological observations to the activation or inhibition of any individual cell population or set of anatomic structures.

Stimulation induces mirth and elevated mood. Dorsal anterior cingulum stimulation elicited a positive affect with immediate smiling and laughing in 3 patients. Concordantly, stimulation at a subjective threshold produced awareness of an internal experience of elevated mood. The patients easily discriminated cingulum stimulation from sham and off-target stimulation conditions, showed insight with respect to their experiences, and provided vivid descriptions of increasing happiness and relaxation in direct correlation to the dose of acute stimulation. In the index patient, prolonged stimulation further altered emotional perceptual processing by inducing a positive shift in bias with respect to the interpretation of images of emotional faces, a validated biomarker associated with enhancement in positive affect $(28,29)$.

Mirthful behaviors, with or without corresponding changes in mood, have previously been described with stimulation of gray matter of the inferior frontal operculum and face motor regions, dorsal ACC, and supplementary motor area (9, 30-32). Relative to previous reports, however, we observed low threshold effects from contacts in the cingulum bundle rather than from contiguous gray matter in the anterior dorsal ACC (contacts 1-2, Figure 1, 
yellow and green). Higher-density stimulation maps of white and gray matter of this region across multiple subjects will ultimately be required to clarify these effects. Our observation that cingulum stimulation produced congruent positive affect and elevated mood may contrast with some reports of mood-incongruent unilateral motor smiling behavior induced at initial stimulation of the ventral internal capsule and ventral striatum (VC/VS) in patients undergoing DBS for obsessive-compulsive disorder $(33,34)$. Our findings also contrast with the observation that VC/VS stimulation can elicit psychomotor activation to the point of hypomania (35). Interestingly, evidence from functional imaging suggests that VC/VS stimulation is only able to improve depression symptoms when it drives increased perfusion of the dorsal ACC (36). Recent instantiations of VC/VS DBS for neuropsychiatric diseases were variably effective $(37,38)$, and it could be hypothesized that more direct and consistent functional engagement of the cingulate region could provide more consistent benefit.

LFP correlates of cingulum stimulation. We observed an endogenous 6- to $11-\mathrm{Hz}$ oscillation recorded in the cingulum bundle, a range that overlaps between generally defined bands of theta (4-7 $\mathrm{Hz}$ ) and alpha $(8-12 \mathrm{~Hz})$ in humans. Indeed, the frequency of oscillations across the other recording contacts varied, such that some (e.g., contacts in hippocampus) showed peak power at approximately 6 to $8 \mathrm{~Hz}$ and others (e.g., contacts in posterior neocortex) showed peak power at approximately 10 to $11 \mathrm{~Hz}$. However, the distribution of peak power frequencies across channels represented a range and was not clearly partitioned into theta or alpha frequency ranges. Thus, we have referred to the oscillations descriptively as $6-$ to $11-\mathrm{Hz}$ oscillations. Indeed, the observation that the electrode contact in the cingulum bundle showed coherence at around $8 \mathrm{~Hz}$ with many of these recording contacts across the brain, suggests that variability of oscillations was best understood as a continuum centered around $8 \mathrm{~Hz}$ rather than discrete bands.

We found that a brief 1-second stimulation was consistently followed by widespread changes in LFP activity from the observed 6- to $11-\mathrm{Hz}$ oscillation baseline. With the caveat that our intracranial electrode coverage favored the dominant (left) hemisphere, changes were notable in the ipsilateral frontal, parietal, and occipital lobes and in the bilateral temporal lobes. Specifically, we observed substantially decreased 6- to $11-\mathrm{Hz}$ power and cingulum coherence across contacts of the lateral and opercular frontalparietal regions and much of the dorsal cingulate region (Brodmann's areas 24 and 33). These changes may have been accentuated by mild background slowing (i.e., prominence of neocortical theta) in our patient at baseline. The apparent involvement of unimodal motor and sensory cortices could have corresponded to motor and behavioral effects, but we did not observe such profound changes with voluntary unstimulated social smiling alone. The stimulation-induced reductions in LFP power and coherence may indicate desynchronization in dorsal ACC and lateral-opercular frontal-parietal regions. In fact, desynchronization of ongoing neural activity has been reported at the onset of many types of cognitive events and has been interpreted by some to reflect reduced activity of the "default mode network" during more externally focused tasks $(39,40)$. Nevertheless, we cannot determine whether the LFP changes we observed were causally related to changes in affect and mood or resulted from more complex effects on attention or arousal networks. Our observations appear to be consistent with those of previous reports linking positive emotionality with reduced alpha activity recorded from scalp EEGs, especially over the dominant hemisphere $(41,42)$.

On the other hand, stimulation was found to induce significant increases in 6- to 11-Hz power in the temporal lobes (most notably in amygdala and parahippocampus), the ipsilateral precuneus, and the ipsilateral occipital lobe. Likewise, increased 6- to 11-Hz coherence was observed between cingulate and the ipsilateral temporal and occipital lobes, but also the ventral ACC, possibly indicating a relative slowing in these networks (Figure 4). The changes we observed in the medial prefrontal, precuneus, and parahippocampal regions are noteworthy, given that CB-II and CB-III carry projections from cingulate cortices to these regions. Given the anxiolytic effect of cingulum stimulation in this case, the increased thetaalpha activity in the amygdala region is additionally notable. This possibly represents a relative emergence of hippocampal thetalike activity, which can occur together with desynchronized neocortical activity in support of arousal and processing of salient and/ or novel input, among other functions $(43,44)$.

Anxiolysis without sedation during an awake craniotomy. Patient anxiety is a common confounder of awake neurosurgical procedures. Even an otherwise insightful and neurologically intact patient can become dysphoric and panic upon emergence from sedation in the unfamiliar and uncomfortable environment of the operating room, when the head is immobilized and the brain is exposed. Unless the patient can be calmed and redirected quickly, sedation must be reintroduced to protect the exposed brain from injury, and the original goals of the operation may be curtailed. By contrast, stimulation in this case induced positive affect and anxiolysis that were clinically meaningful and nonsedating. Escalating stimulation immediately enhanced the patient's comfort, allowed complete withdrawal of intravenous sedating anesthetic medication, and did not interfere with cognitive performance. When stimulation was unintentionally withdrawn, our patient eventually resumed a state of dysphoria, becoming tearful and afraid. Resumption of stimulation, rather than anesthetic sedation, again provided immediate anxiolysis, and the goals of the operation (maximal safe resection) were achieved, further underlining the utility of stimulation in this case.

Limitations and future directions. In the index patient, our tractographic methods demonstrated that anxiolytic stimulation (contacts 3-4) produced near-perfect engagement of the cingulum bundle, to the exclusion of nearly all other fiber systems (the exception possibly being minor engagement of the superior aspect of the corpus callosum at a higher electrical current). By comparison, VTA models of the anterior (primarily cingulate gray matter, contacts 1-2) pair probably produced only limited engagement of the cingulum bundle and were not associated with any patient-reported subjective effects. VTA models of the posterior cingulum bundle pair (contacts 5-6) were associated with robust engagement not only of the cingulum bundle but also of the superior aspect of the corpus callosum innervating primary motor and sensory cortices. This pattern of connectivity was notable, given that this contact pair evoked mirth as well as motor activation. One limitation of this aspect of the study is that our VTA models are based on electrodynamic models of monopolar stimulation 
delivered in the context of DBS, in which the electrode diameter is larger and the contact spacing is narrower than in the current case. A future direction of the current research includes improved VTA modeling of distinct electrode geometries and configurations. The remarkably similar impact of acute cingulum bundle stimulation in our small series of 3 patients with epilepsy (index female patient, left cingulum; male patient 2, right cingulum; and female patient 3 , right cingulum) with distinct seizure-onset zones suggests that the effects we observed were not idiosyncratic, highly lateralizing, or sex specific. Further, in terms of neural reorganization in epilepsy, recent work has shown that the impact on large-scale network organization may be much smaller than previously assumed (45). Sex differences have also been noted in cingulum bundle microstructure, in which men are noted to have higher fractional anisotropy (a marker of myelination) than women, suggesting that future work must further address functional neuroanatomic differences between the sexes. The current study used a relatively limited range of stimulation parameters similar to those previously reported (11), but future studies will benefit from exploration of a wider-parameter space, such as the effects of more physiological frequencies of stimulation. Future studies may also explore the mechanisms of anxiolysis and the potential of generalization to primary anxiety disorders by comparing resting-state functional MRI (fMRI) networks of the current patient with those of healthy controls, patients with primary anxiety disorders, and other epilepsy patients.

Clinical implementation. Our initial observations suggest that acute cingulum stimulation is a novel, readily translatable approach to managing patient anxiety during awake neurosurgical procedures. Unlike currently accepted DBS targets in gray matter (which can be reduced to recommended average coordinate locations), the therapeutic target we have explored is a white matter fiber bundle in the frontal lobe that is well known to show significant interindividual variability. Therefore, the future approach for targeting this region may rely on individual patient diffusion tensor imaging and the purposeful positioning of contacts to be centrally located in the bundle, such that bipolar stimulation may be passed parallel to the bundle. Our data imperfectly sample the anterior-posterior extent of the bundle, but we have seen the greatest efficacy from the anterior-dorsal portion of the bundle. More specifically, all behaviorally active contacts were localized to an approximately $1-\mathrm{cm}$ span directly superior to the interface of the genu of the corpus callosum with the lateral ventricle from the parasagittal plane of the cingulum bundle. Thus, to implement cingulum stimulation more broadly, patient-specific targeting will rely on diffusion MRI-based tractography and electrophysiological profiling to validate target engagement. As with any novel brain stimulation target, a systematic approach to establishing general and patient-specific stimulation parameters for greatest efficacy with minimal side effects will be required. The current observational study must be followed up with a formal experimental trial or phase I clinical trial in order to establish the safety and efficacy of optimal stimulation.

We believe our report has direct implications for the development of new therapies for anxiety disorders such as panic disorder, posttraumatic stress disorder, and obsessive-compulsive disorder. After exhausting first-line medications and psychologi- cal interventions, patients with severe anxiety disorders are often treated with benzodiazepines and barbiturates (46), which cause sedation, impair cognitive function (47), and carry a high risk of dependence and abuse (48). If anxiolysis is found in additional patients to be robust and consistent, DBS of the cingulum bundle may offer a novel alternative for treating severe anxiety disorders.

\section{Methods}

Setting. Research stimulation was completed in the epilepsy monitoring unit, an inpatient unit with 24-hour video EEG surveillance and nursing support, and clinical stimulation was applied in the neurosurgical operating room. The patients underwent implantation of iEEG electrodes (grids and strips of 5-mm diameter, 10-mm spacing; depth arrays $0.86-\mathrm{mm}$ diameter; 2-mm length platinum-plated contacts, spaced at 5- to 8-mm intervals; AdTech Medical Instrument Corp.). Electrodes were placed to interrogate the hypothesized seizure-onset zones and their networks. Research study inclusion required the patients to be English-speaking adults ( $>18$ years, regardless of sex, race, or ethnicity), implanted with intracranial depth electrodes. The patients were able to converse and respond to questions about their subjective feelings.

Stimulation. Electrical stimulation and passive neural recording took place via implanted electrodes that spanned the longitudinal extent of the cingulum bundle (Figure 1D). Research stimulation was generated by a Blackrock Microsystems CereStim R96 neural stimulation device with Blackrock Stim Manager Software. Stimulation parameters included frequencies of $130 \mathrm{~Hz}, 300$ - $\mu$ s pulse width (alternately $50 \mathrm{~Hz}, 200-\mu$ s pulse width) from 0.5 to $3.5 \mathrm{~mA}$ using a bipolar montage and a charge-balanced biphasic square waveform, delivered to electrode contacts (surface area $0.059 \mathrm{~cm}$; impedance 1-4 $\mathrm{k} \Omega$ ). The impedances of the AdTech reduced-diameter depth electrodes in the human brain measured at approximately $850 \pm 100 \mathrm{Ohms}$ in a separate patient undergoing resonance frequency ablation; because of theoretical concerns that impedance checking could harm the tissue, these values were checked only in locations that were targets for destructive lesions. No after-discharges were noted with stimulation to the cingulum bundle. Cingulum stimulation in the operating room was delivered via a Medtronic DualStim 3628 hand-held, voltage-controlled neural stimulator in $0.5-\mathrm{V}$ steps from $0.5 \mathrm{~V}$ to $4.5 \mathrm{~V}$ intraoperatively, after immediate preoperative screening indicated overall analogous thresholds for behavioral response when rendered in volts (approximately $2 \mathrm{~V}$ ) and $\mathrm{mA}$ (approximately $1.5 \mathrm{~mA}$ ). Our method for the selection of stimulation amplitudes involved an empiric balance between the observation of thresholds for behavioral effects, avoiding electrophysiological after-discharges at increasing amplitudes and remaining below a $30-\mu \mathrm{C} / \mathrm{cm}^{2}$ charge density.

Affective bias task and machine-learning analysis of the subject's facial expressions. The affective bias task asks participants to rate the valence and intensity of facial emotional expressions using a visual analog scale. Stimuli are adapted from the Macarthur face stimulus set, including 3 female and 3 male identities (49). Stimuli began as happy, neutral, and sad exemplars, from which morphs were interpolated using a Delaunay tessellation matrix to generate subtle facial expressions. The final stimulus set included 100\%, 50\%, 30\%, and $10 \%$ happy and sad faces as well as neutral faces. The task shows high within-subjects test-retest reliability $(r=0.903)$ as compared with measures of self-reported mood $(r=0.579)$ (50). The patient completed 
the task in blocked form, in which all faces ranging from neutral to sad were presented in randomized order within a block, and all faces ranging from neutral to happy were presented randomized within another block. These sets of blocks (one sad, one happy) were repeated 3 times on research day 1 (sham; dorsal anterior cingulate gray matter $50 \mathrm{~Hz}$, $3.5 \mathrm{~mA}, 200-\mu \mathrm{s}$ stimulation; dorsal anterior cingulate gray matter 130 $\mathrm{Hz}, 3.5 \mathrm{~mA}, 300-\mu \mathrm{s}$ stimulation) (Figure 1), and 3 times on research day 2 (sham; 0.5-mA stimulation of the dorsal cingulum bundle; 1.0-mA stimulation of the dorsal cingulum bundle) (Figure 1). Data for the day-one $50-\mathrm{Hz}$ stimulation and day-two sham stimulation were excluded from the analysis for the sake of clarity. Day-2 sham data were interpreted to be contaminated, as they reflected a significant positive shift in affective biasing and followed 10 minutes after approximately 90 minutes of active cingulum stimulation testing, during which the patient had been laughing frequently. The appropriate washout period of the cingulum stimulation effect is unknown, but this washout phenomenon was again observed following prolonged stimulation during awake surgery, in which the patient's mood remained elevated for several minutes after unintentional discontinuation.

Continuous video recording was collected during each run of the bias task, and machine learning was applied by fitting a linear dynamical system to the video to identify segments with significant clustering of intensity and dynamics over time and comparing each segment in terms of their angular distance in a Euclidian space (25). The comparison of segments was repeated for each possible pair, giving a similarity matrix. Modes of pixel-wise intensity dynamical clustering during video recording of the patient's face during the affective bias task were found to align with periods of time in which she was completing different blocks of the task (evaluating happy vs. sad faces) under different stimulation conditions (sham, subthreshold cingulum stimulation, suprathreshold cingulum stimulation).

Imaging. High-resolution T1 MRI (1.5T Avanto, Siemens Medical Solutions) and CT (LightSpeed 16, GE Medical Systems) images were collected preoperatively, and diffusion-weighted MRI (3T Magnetom Prisma, Siemens Medical Solutions) images were acquired after the explantation of depth electrodes and responsive neural stimulation equipment. Preoperative T1: resolution $=1 \times 1 \times 1 \mathrm{~mm}^{3}$, repetition time/ echo time $(\mathrm{TR} / \mathrm{TE})=1900 / 3.52 \mathrm{~ms}$, matrix $=176 \times 198 \times 198$; post-depth T1: resolution $=1 \times 1 \times 1 \mathrm{~mm}^{3}$, TR/TE $=1900 / 3.5 \mathrm{~ms}$, matrix $=206 \times 249 \times$ 176 ; CT: resolution $=1 \times 1 \times 1$, matrix $=256 \times 256 \times 175$; postoperative $3 \mathrm{~T}$ multishell diffusion-weighted imaging (DWI) using a multiband accelerated echo planar sequence: resolution $=1.75 \times 1.75 \times 1.75 \mathrm{~mm}^{3}$, TR/ $\mathrm{TE}=3430 / 90 \mathrm{~ms}$, acquisition matrix $=128 \times 128 \times 80,60$ noncollinear directions with 11 nondiffusion-weighted images $(b=0)$, two opposite phase-encoding directions (anterior-posterior and posterior-anterior) to compensate for susceptibility-induced distortion artifacts, multishell acquisitions $\mathrm{b}$ value $=1000$ and $2000 \mathrm{~s} / \mathrm{mm}^{3}$, multiband accelerator factor $=3$. All image processing and analyses were performed using tools from the FMRIB Software Library (FSL) (http://fsl.fmrib.ox.ac. $\mathrm{uk} / \mathrm{fsl} / \mathrm{fslwiki} /$ ) and TrackVis (trackvis.org). Seed volumes were derived from "volume of tissue activated" modeling $(14,20)$.

Autonomic methods. During stimulation parameter testing, autonomic data were collected to quantify palmar electrodermal activity (EDA) (i.e., skin conductance), heart rate, and pulse photoplethysmography, using a Biopac MP150 unit with Bionomadix wireless transmission modules (BIOPAC Systems). Stimulation was applied in 30-second increments while data were recorded and analyzed in Acq- knowledge 4.2. The heart rate was calculated from the ECG data. All artifacts were manually cleaned using Acqknowledge 4.2 software by linearly interpolating across the artifactual data. EDA (skin conductance response) amplitude was calculated as the magnitude of the peak-to-peak change in EDA from stimulation onset to offset and in two 15-second intervals during stimulation. Average changes in heart rate were calculated after subtracting a 15 -second prestimulus baseline for the 30-second stimulation periods and in 15-second windows across the stimulation periods (Supplemental Figure 4).

Electrophysiology methods. LFPs (DC-1000 Hz signals) were recorded with FDA- and Emory University IRB-approved devices in the Emory Epilepsy Monitoring Unit, using the XLTEK Natus NeuroLink IP integrated clinical and research EEG data acquisition system (Natus Medical). Electrophysiological data were directly uploaded to a secure 2-petabyte RAID server securely managed by Emory Healthcare. Data were recorded from 127 channels, including 4 surface grids, 8 surface strips, and 12 depth arrays implanted strategically to maximize the likelihood that the patient's seizure focus could be adequately localized. These data were collected continuously but were specifically analyzed during a transient stimulation experiment, in which stimulation was applied for 1 second at approximately 11-second intervals, interleaving active and sham stimulation conditions for a total of 30 exposures (20 active stimulations, 10 sham stimulations). Analyses were performed using the Chronux Toolbox for MATLAB for spectral analyses to estimate power and coherence by comparing the 3 seconds preceding stimulation onset to the 3 seconds after the offset of stimulation to avoid confounding stimulation artifacts and to examine network correlates of the persistent improvement in affect following each active stimulation (51).

Statistics. Statistical analyses conducted for data from a single patient are best considered a descriptive metric of strength of associations. Analysis of cingulum local electrophysiological impacts uses error bars showing 95\% CIs was generated by a random reshuffling procedure. For each channel, prestimulation, poststimulation, and sham data were swapped on a trial-by-trial basis. Then, the mean power/ coherence difference (poststimulation minus prestimulation) for this randomly shuffled data were calculated. The procedure was repeated a total of 1000 times (sampling with replacement from possible shuffles), thus generating a distribution of differences that reflected the null hypothesis. The actual difference between prestimulation and poststimulation power in the cingulum bundle contact was $-1.76 \mathrm{~dB}$. The inner 95th percentile of the random distribution ranged from $-1.33 \mathrm{~dB}$ to $+1.33 \mathrm{~dB}$. Thus, the actual difference was more extreme than most of the randomly generated differences, or the $P$ value was less than 0.05 . Network analysis: $P$ values were calculated for each electrode for power (stim-baseline) and coherence (stim-baseline). In each case, the $P$ value reflects the percentile of the null (random) distribution at which the actual value was observed (rendered in Figure 4 with a color heatmap). The overall effect was evaluated using basic probability (binomial probability modeled using "binopdf" function in MATLAB 2017a (MathWorks). For heart rate analyses, we used standard calculations of the mean and SDs on the basis of recorded ECG and bpm.

Study approval. Our research study (extraoperative limbic stimulation with behavioral, electrophysiological, autonomic, and neuroimaging analysis during routine intracranial monitoring for epilepsy) was approved by the IRB of Emory University (IRB no. 00067252) and conducted in accordance with Declaration of Helsinki regulations. To avoid the possibility of perceived coercion, recruitment to the study 
and informed consent were handled by nonclinical study staff rather than the patient's own physician. In this case, written informed consent for all participants was obtained by KRB. The application of stimulation for anxiolysis during craniotomy in the index patient took place on clinical grounds as determined by consensus of the treating physicians (anesthesiologist, neurologist, neurosurgeon, and clinical neuropsychologist), with clinical consent from the patient and support in this decision from her family members. Written informed consent was provided by the index patient for the use of her deidentified photographs and video recordings. All patients provided separate informed consent for surgical and research-related procedures.

\section{Author contributions}

KRB, HSM, DLD, and JTW conceived the study. KRB, CSI, NPP, and JTW designed the study methodology. KRB, JRM, KSC, and $\mathrm{SH}$ performed the formal analysis. KRB, CSI, NPP, REF, and JTW performed experiments. KRB and JTW wrote the original draft of the manuscript. KRB, JTW, JRM, REF, KSC, CSI, NPP, ACW, HSM, DLD, and SH wrote, edited, and reviewed the manuscript. JTW, HSM, and DLD supervised the study.

\section{Acknowledgments}

We would like to thank the following individuals for their significant contributions to the development of this article: John Gale, Robert Gross, Grant Lynde, Joel Zivot, Camille Steger, and Taylor Shade of the Emory University School of Medicine. We would also like to thank the patients for participating in the current study. This project was supported by the American Foundation for Suicide Prevention (YIG-0-015-13, to KRB); the National Center for Advancing Translational Sciences (NCATS), NIH (UL1TR002378 and KL2TR002381, to KRB); the National Institute of Neurological Disorders and Stroke (NINDS), NIH (R21NS104953, to KRB and JTW; K08NS105929, to NPP; and R01NS088748, K02NS070960, to DLD); and the National Institute of Mental Health (NIMH), NIH (K01MH116364, to KRB). The content is solely the responsibility of the authors and does not necessarily represent the official views of the NIH.

Address correspondence to: Jon T. Willie, 1365 Clifton Road NE, Suite B6200, Atlanta, Georgia 30322, USA. Phone: 404.727.2354; Email:jon.t.willie@emory.edu.
1. Wu Y, Sun D, Wang Y, Wang Y, Ou S. Segmentation of the cingulum bundle in the human brain: A aew perspective based on DSI tractography and fiber dissection study. Front Neuroanat 2016;10:84.

2. Jones DK, Christiansen KF, Chapman RJ, Aggleton JP. Distinct subdivisions of the cingulum bundle revealed by diffusion MRI fibre tracking: implications for neuropsychological investigations. Neuropsychologia. 2013;51(1):67-78.

3. Vogt BA, Pandya DN. Cingulate cortex of the rhesus monkey: II. Cortical afferents. JComp Neurol. 1987;262(2):271-289.

4. Cohen MX, Heller AS, Ranganath C. Functional connectivity with anterior cingulate and orbitofrontal cortices during decision-making. Brain Res Cogn Brain Res. 2005;23(1):61-70.

5. Parvizi J, Rangarajan V, Shirer WR, Desai N, Greicius MD. The will to persevere induced by electrical stimulation of the human cingulate gyrus. Neuron. 2013;80(6):1359-1367.

6. Fossati P. Neural correlates of emotion processing: from emotional to social brain. Eur Neuropsychopharmacol. 2012;22 Suppl 3:S487-S491.

7. Jahn A, Nee DE, Alexander WH, Brown JW. Distinct regions of anterior cingulate cortex signal prediction and outcome evaluation. Neuroimage. 2014;95:80-89.

8. Sperli F, Spinelli L, Pollo C, Seeck M. Contralateral smile and laughter, but no mirth, induced by electrical stimulation of the cingulate cortex. Epilepsia. 2006;47(2):440-443.

9. Caruana F, Avanzini P, Gozzo F, Francione S, Cardinale F, Rizzolatti G. Mirth and laughter elicited by electrical stimulation of the human anterior cingulate cortex. Cortex. 2015;71:323-331.

10. Russo JF, Sheth SA. Deep brain stimulation of the dorsal anterior cingulate cortex for the treatment of chronic neuropathic pain. Neurosurg Focus. 2015;38(6):E11.

11. Boccard SG, et al. Targeting the affective component of chronic pain: a case series of deep brain stimulation of the anterior cingulate cortex. Neurosurgery. 2014;74(6):628-635.

12. Spooner J, Yu H, Kao C, Sillay K, Konrad P. Neuromodulation of the cingulum for neuropathic pain after spinal cord injury. Case report. J Neurosurg. 2007;107(1):169-172.

13. Riva-Posse $\mathrm{P}$, et al. A connectomic approach for subcallosal cingulate deep brain stimulation surgery: prospective targeting in treatment-resistant depression. Mol Psychiatry. 2018;23(4):843-849.

14. Riva-Posse P, et al. Defining critical white matter pathways mediating successful subcallosal cingulate deep brain stimulation for treatment-resistant depression. Biol Psychiatry. 2014;76(12):963-969.

15. Lozano AM, et al. A multicenter pilot study of subcallosal cingulate area deep brain stimulation for treatment-resistant depression. J Neurosurg. 2012;116(2):315-322.

16. Lozano AM, et al. A phase II study of fornix deep brain stimulation in mild Alzheimer's disease. J Alzheimers Dis. 2016;54(2):777-787.

17. Bewernick BH, Kayser S, Gippert SM, Switala C, Coenen VA, Schlaepfer TE. Deep brain stimulation to the medial forebrain bundle for depression- long-term outcomes and a novel data analysis strategy. Brain Stimul. 2017;10(3):664-671.

18. Cossu M, et al. Stereoelectroencephalography in the presurgical evaluation of children with drug-resistant focal epilepsy. J Neurosurg. 2005;103(4 Suppl):333-343.

19. Beck AT, Ward CH, Mendelson M, Mock J, Erbaugh J. An inventory for measuring depression. Arch Gen Psychiatry. 1961;4:561-571.

20. Chaturvedi A, Luján JL, McIntyre CC. Artificial neural network based characterization of the volume of tissue activated during deep brain stimulation. J Neural Eng. 2013;10(5):056023.

21. Trzepacz PT, Baker RW. The Psychiatric Mental Status Examination. Oxford, UK: Oxford University Press; 1993.

22. Bouhuys AL, Geerts E, Gordijn MC. Depressed patients' perceptions of facial emotions in depressed and remitted states are associated with relapse: a longitudinal study. J Nerv Ment Dis. 1999;187(10):595-602.

23. Gur RC, Erwin RJ, Gur RE, Zwil AS, Heimberg C, Kraemer HC. Facial emotion discrimination: II. Behavioral findings in depression. Psychiatry Res. 1992;42(3):241-251.

24. Surguladze SA, Young AW, Senior C, Brébion G, Travis MJ, Phillips ML. Recognition accuracy and response bias to happy and sad facial expressions in patients with major depression. Neuropsychology. 2004;18(2):212-218.

25. Harati S, Crowell A, Mayberg H, Jun K, Nemati S. Discriminating clinical phases of recovery from major depressive disorder using the dynamics of facial expression. Conf Proc IEEE Eng Med Biol Soc. 2016;2016:2254-2257.

26. Ranck JB. Which elements are excited in electriwcal stimulation of mammalian central nervous system: a review. Brain Res. 1975;98(3):417-440.

27. McIntyre CC, Savasta M, Kerkerian-Le Goff L, Vitek JL. Uncovering the mechanism(s) of action of deep brain stimulation: activation, inhibition, or both. Clin Neurophysiol. 2004;115(6):1239-1248.

28. Harmer CJ, et al. Effect of acute antidepressant administration on negative affective bias in depressed patients. Am J Psychiatry. 2009;166(10):1178-1184.

29. Godlewska BR, Browning M, Norbury R, Cowen PJ, Harmer CJ. Early changes in emotional processing as a marker of clinical response to SSRI treatment in depression. Transl Psychiatry. 2016;6(11):e957.

30. Fernández-Baca Vaca G, Lüders HO, Basha MM, Miller JP. Mirth and laughter elicited during brain stimulation. Epileptic Disord. 2011;13(4):435-440.

31. Krolak-Salmon P, et al. Experiencing and detecting happiness in humans: the role of the supplementary motor area. Ann Neurol. 2006;59(1):196-199. 
32. Caruana F, Gozzo F, Pelliccia V, Cossu M, Avanzini P. Smile and laughter elicited by electrical stimulation of the frontal operculum. Neuropsychologia. 2016;89:364-370.

33. Haq IU, et al. Smile and laughter induction and intraoperative predictors of response to deep brain stimulation for obsessive-compulsive disorder. Neuroimage. 2011;54(Suppl 1):S247-S255.

34. Okun MS, et al. What's in a "smile?" Intraoperative observations of contralateral smiles induced by deep brain stimulation. Neurocase. 2004;10(4):271-279.

35. Haq IU, et al. A case of mania following deep brain stimulation for obsessive compulsive disorder. Stereotact Funct Neurosurg. 2010;88(5):322-328.

36. Dougherty DD, et al. Acute deep brain stimulation changes in regional cerebral blood flow in obsessive-compulsive disorder. J Neurosurg. 2016;125(5):1087-1093.

37. Dougherty DD, et al. A randomized shamcontrolled trial of deep brain stimulation of the ventral capsule/ventral striatum for chronic treatment-resistant depression. Biol Psychiatry. 2015;78(4):240-248.

38. Greenberg BD, et al. Deep brain stimulation of the ventral internal capsule/ventral striatum for obsessive-compulsive disorder: worldwide experience. Mol Psychiatry. 2010;15(1):64-79.

39. Buckner RL. The serendipitous discovery of the brain's default network. Neuroimage. 2012;62(2):1137-1145.

40. Raichle ME, MacLeod AM, Snyder AZ, Powers WJ, Gusnard DA, Shulman GL. A default mode of brain function. Proc Natl Acad Sci U S A. 2001;98(2):676-682.

41. Vytal K, Hamann S. Neuroimaging support for discrete neural correlates of basic emotions: a voxel-based meta-analysis. J Cogn Neurosci. 2010;22(12):2864-2885.

42. Davidson RJ, Ekman P, Saron CD, Senulis JA, Friesen WV. Approach-withdrawal and cerebral asymmetry: emotional expression and brain physiology. I. J Pers Soc Psychol. 1990;58(2):330-341.

43. Buzsáki G, Moser EI. Memory, navigation and theta rhythm in the hippocampal-entorhinal system. Nat Neurosci. 2013;16(2):130-138.

44. Tesche CD, Karhu J. Theta oscillations index human hippocampal activation during a working memory task. Proc Natl Acad Sci U S A. 2000;97(2):919-924.

45. Corina DP, Loudermilk BC, Detwiler L, Martin RF, Brinkley JF, Ojemann G. Analysis of naming errors during cortical stimulation mapping: implications for models of language representation. Brain Lang. 2010;115(2):101-112.

46. Cloos JM, Ferreira V. Current use of benzodiazepines in anxiety disorders. Curr Opin Psychiatry. 2009;22(1):90-95.

47. Curran HV, Collins R, Fletcher S, Kee SC, Woods B, Iliffe S. Older adults and withdrawal from benzodiazepine hypnotics in general practice: effects on cognitive function, sleep, mood and quality of life. Psychol Med. 2003;33(7):1223-1237.

48. Olfson M, King M, Schoenbaum M. Benzodiazepine use in the United States. JAMA Psychiatry. 2015;72(2):136-142.

49. Tottenham N, et al. The NimStim set of facial expressions: judgments from untrained research participants. Psychiatry Res. 2009;168(3):242-249.

50. Bijanki KR, et al. Case report: stimulation of the right amygdala induces transient changes in affective bias. Brain Stimul. 2014;7(5):690-693.

51. Bokil H, Andrews P, Kulkarni JE, Mehta S, Mitra PP. Chronux: a platform for analyzing neural signals. J Neurosci Methods. 2010;192(1):146-151.

52. Mai JK, Paxinos G, Voss T. Atlas of the Human Brain. 3rd ed. San Diego, California, USA: Academic Press; 2008. 